International Journal of Case Reports
(ISSN:2572-8776)

\title{
The Rare Case of Thromboangiitis Obliterans in a Patient Previously Diagnosed with a Stroke
}

\section{Niki Parikh and Franklyn Babb}

Texas Tech University Health Sciences Center, Department of Family Medicine, 3601 4th Street, STOP 8312, Lubbock, TX 79430-8312

\section{ABSTRACT}

Thromboangiitis obliterans (Buerger's disease) is a disease of small and medium-sized vasculature, mainly involving the dis${ }^{*}$ Correspondence to Author: tal upper and lower extremities in male patients under the age Niki Parikh, MBA, MSBA of 45. Smoking tobacco is considered to be a predisposing and Texas Tech University Health Scitriggering agent. This presentation discusses the case of JS, a ly Medicine, 36014 th Street, STOP 35-year old male patient who initially presented with a stroke, 8312, Lubbock, TX 79430-8312 followed by pain and swelling in the upper extremity, and finally with pain, absent peripheral pulses, and necrosis of the left lower extremity requiring below knee amputation. This case is of interest due to the rarity of this disease in the developed world, the How to cite this article:

presentation of a stroke in a young patient later diagnosed with thromboangiitis obliterans, and the use of a prostaglandin analog in treatment. This case highlights the difficulty of making the diagnosis of vasculitis and the time consuming, multispecialty approach that is required. The availability of subspecialists, medical record integration, communication between community hospitals, and the drug lloprost could have positively affected the patient's outcomes and prognosis.

Keywords: thromboangiitis obliterans, buerger, winiwarter-buergNiki Parikh and Franklyn Babb. The Rare Case of Thromboangiitis Obliterans in a Patient Previously Diagnosed with a Stroke. International Journal of Case Reports, 2019 4:71 er, iloprost, smoking

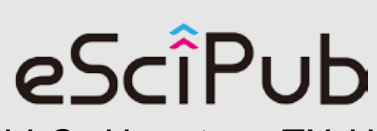

eSciPub LLC, Houston, TX USA. Website: http://escipub.com/ 


\section{Introduction}

Buerger's disease, also known as WiniwarterBuerger disease or thromboangiitis obliterans (TAO), is a non-atherosclerotic, inflammatory vasculitis of small and medium-sized vasculature most commonly seen in young men with a history of smoking tobacco. ${ }^{1}$ The disease is most prevalent in the Mediterranean, Middle East, and Asia, where tobacco use is prevalent. Clinical presentation involves migratory thrombophlebitis, gangrene, autoamputation of the upper and lower extremities, and Raynaud's phenomenon. Cerebral involvement is rare. The disease is a diagnosis of exclusion by elimination of other causes of vasculitis. This report discusses a recently diagnosed case of stroke and limb ischemia in a TAO patient at a tertiary care facility in Texas.

\section{Case Presentation}

A 35-year-old male with a past medical history of hypertension, asthma, alcohol abuse, marijuana use, and 46-pack-year smoking history presented to the emergency room complaining of numbness and weakness in his right upper and lower extremities. Neurological examination revealed diminished pin-prick sensation, 4/5 motor tone and power on the right upper and lower extremities, and hyperreflexia on the right upper and lower extremities. CT scan revealed no abnormalities; however, MRI showed a left pontine infarction with absence of intracranial bleeding or other vascular malformations (Figure 1). A full work-up was conducted to determine etiology of the stroke, however no identifiable cause was noted. The patient's symptoms resolved with complete recovery of neurological function.

Three months later, the patient was admitted to the emergency department with throbbing pain, weakness, and numbness in his right upper extremity, which resolved in 24 hours. Once again, no identifiable causes were discovered, and a thrombo-embolic source was excluded. The patient was readmitted approximately one year later with similar symptoms in his right lower extremity. He claimed he had woken up with cold toes that progressed to involve his entire right foot extending up to the ankle. The patient described the pain as sharp, an 8/10 in severity, and worsening throughout the day with associated weakness and numbness. Physical examination revealed cold and absent peripheral pulses in the dorsalis pedis and posterior tibial arteries. The skin was intact with no ulcerations present.

Vascular ultrasound was conducted, which demonstrated occlusion at the right mid-calf. CT angiogram was then carried out to determine if revascularization was an option; opacification of the peroneal artery to the distal foreleg was detected with no opacification noted in the anterior or posterior tibial arteries below the midcalf. Further study into the imaging revealed the appearance of corkscrew vessels, which is a feature of thromboangiitis obliterans (Figure 2). Due to the lack of rheumatology specialists at the local hospital, the patient was not started on appropriate therapy until he was transferred to a tertiary care center where rheumatology, cardiology and surgery were consulted. The patient was placed on heparin, nicardipine, hydrocodone/paracetamol, morphine, hydromorphone, gabapentin, treprostinil, and varenicline. Over time, the patient developed rhabdomyolysis and a decision was made to perform a below-the-knee amputation one month later. Pathological examination was performed on the amputated tissue and revealed acute cellulitis with fat necrosis, minimal atherosclerosis of blood vessels, and inflammatory vasculopathy, consistent with thromboangiitis obliterans.

\section{Discussion}

Educational efforts by the media as well as the awareness raised by the medical community has caused tobacco use in the United States to decline. This has led to a decrease in the incidence of thromboangiitis obliterans from 104 cases per 100,000 population in $1947^{2}$ to an estimated $12.6-20$ cases per 100,000 population currently. ${ }^{3}$ Only twelve patients have been 


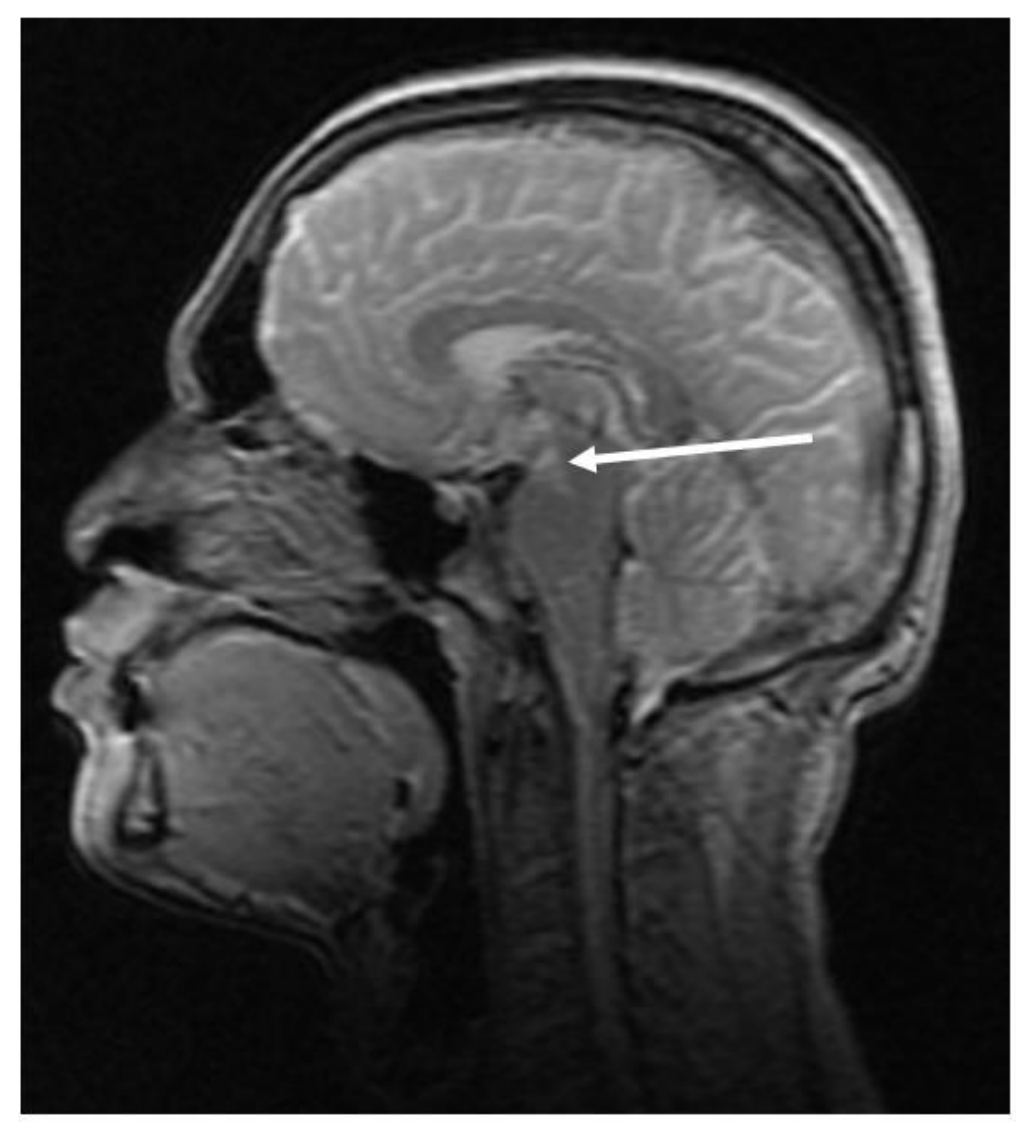

Figure 1: MRI showing left pontine infarction with absence of intracranial bleeding or other vascular malformations

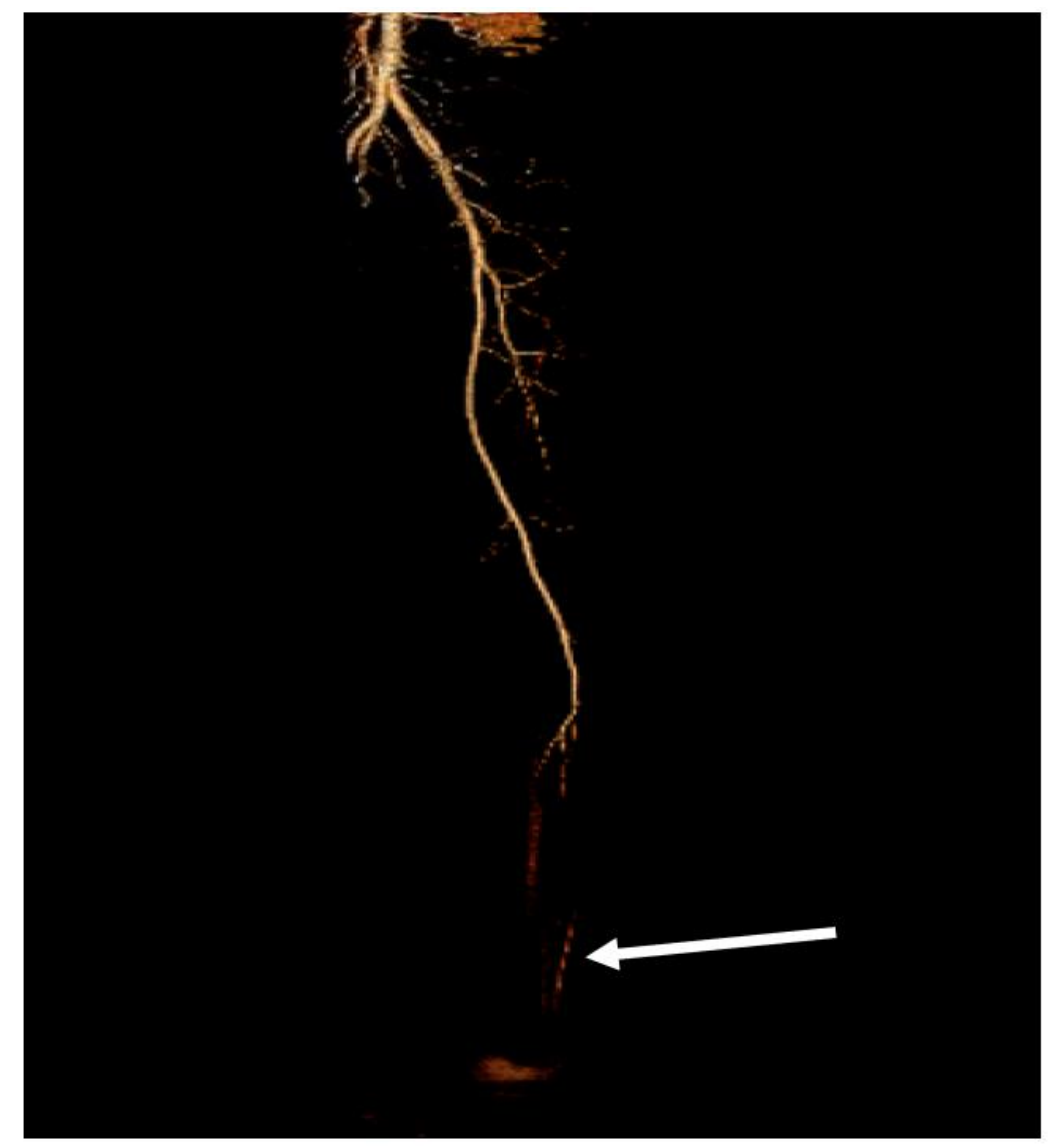

Figure 2: CT angiogram showing opacification of the peroneal artery to the distal foreleg. Arrow demonstrates the appearance of corkscrew vessels, a unique finding of TAO. 
diagnosed with the disease from 2012 to 2016 at the same tertiary care center in Texas.

Due to the rarity of the disease as well as the illdefined pathogenesis, targeted treatments have not yet been discovered. Currently, the only proven treatment for TAO is smoking cessation, which has been shown to reverse or prevent the progression of the disease. ${ }^{4}$ Other pharmacologic agents such as high-potency vasodilators have been shown to reduce the risk of amputation, but lloprost, a $\mathrm{PGl}_{2}$ analogue, is the only one shown to be effective in clinical trials. ${ }^{5}$ This may largely be due to its action in preventing cell proliferation, decreasing platelet aggregation, and causing vasodilation of blood vessels. Other studies have shown that lloprost is also effective for eradicating resting pain and promoting the healing of ischemic ulcers. ${ }^{6}$ While the use of lloprost would have been preferred in this situation, it is very expensive and difficult to obtain. Pharmacies in the local area were contacted to determine the availability of the drug and it was reported that the lack of inventory is due to cost, shelf life, and expiratory date related waste. For this reason, Treprostinil was used for this patient. One published case reported success with the drug in a refractory case of TAO in a patient with critical limb ischemia, however larger multicenter clinical trials are needed to demonstrate its efficacy. ${ }^{7}$

This case is also unique due to the presentation of a stroke, potentially caused by TAO, in this patient. Cerebrovascular thromboangiitis obliterans is often a controversial topic due to the lack of diagnostic evidence supporting the origin of the disease. Patients with TAO have many of the comorbidities seen in patients presenting with a stroke due to other vascular causes, such as hypertension, diabetes, and a smoking history ${ }^{8}$, however it is a topic that should be pursued further. Neurologic signs have been shown to occur in fewer than $2 \%$ of TAO cases and occur most often on the cortical surface and adjacent territories. ${ }^{9}$ A literature review was conducted by Lippmann examining the case study of 39 patients diagnosed with cerebrovascular thromboangiitis obliterans. ${ }^{10} \mathrm{He}$ described two presentations commonly seen with the disease: 1) emotional disturbances and aphasia from thromboendarteritis of the anterior, medial, and posterior cerebral arteries and 2) cortical signs and symptoms from thrombosis of larger brain arteries. Smoking was shown to be a trigger for the disease and patients experienced recurrences and remissions of the focal cortical signs, similar to the remission our patient experienced. Other cases have discussed systematic spread of the disease, including cerebral, mesenteric, cardiac, hepatic, and peripheral vessel involvement. ${ }^{11}$ Currently, cessation of smoking is the only known treatment for the systemic condition.

Lastly, this case demonstrates the importance of a multidisciplinary team approach. The involvement of a team consisting of rheumatology, cardiology, vascular surgery, hematology, radiology family medicine, pain management, and pharmacists was essential in prompt diagnosis and treatment. Our case illustrates the importance of family medicine hospitalists in coordinating the care between various specialists, but it also exposes the vulnerabilities of our health care system. The lack of communication between community hospitals, lack of integration between electronic medical records, and the lack of appropriate physician personnel at hospitals poorly impacted our patient's prognosis. Due to the absence of rheumatology at the local rural hospital, the patient had to be transferred to a tertiary hospital. This delay as well as a delay in communication and obtainment of resources resulted in a setback in obtaining Treprostinil. These were preventable factors and should be noted for future consideration.

\section{Conclusion}

Buerger's disease remains to be a difficult disease to diagnose and the patient must be subjected to numerous tests to rule out other more common causes of ischemia. This is a very time-consuming process of elimination, which often leads to a great time lapse between 
diagnosis and treatment, making the disease irreversible. Cessation of smoking has thus far been the most effective way to stop progression of this disease and a multidisciplinary approach is needed to benefit the patient. A doubleblinded control study is needed to prove efficacy of Treprostinil and lloprost before determining its viability in patients with thromboangiitis obliterans disease.

Financial Support: no grants or financial support received

Conflict of Interest: authors have no conflict of interest

\section{References}

1 Zerbino DD, Zimba EA, Bagry NN. [Thromboangiitis obliterans (Buerger's disease): state of the art]. Angiol Sosud Khir. 2016; 22: 185-92.

2 Aqel M, Olin J. Thromboangiitis Obliterans (Buerger's Disease). Sage Journals. 1997; 2.

3 Buerger's Disease. Rare Disease Database. National Organization for Rare Disorders 2007.

4 Fiessinger JN, Frank M. [THROMBOANGIITIS OBLITERANS (BUERGER'S DISEASE)]. Rev Prat. 2015; 65: 1079-83.
5 Jiménez-Gallo D, Albarrán-Planelles C, ArjonaAguilera C, Blanco-Sánchez G, Rodríguez-Mateos $M E$, Linares-Barrios M. Treatment of thromboangiitis obliterans (Buerger's disease) with high-potency vasodilators. Dermatol Ther. 2015; 28: 135-9.

6 Cacione DG, Macedo CR, Baptista-Silva JC. Pharmacological treatment for Buerger's disease. Cochrane Database Syst Rev. 2016; 3: CD011033.

7 Fernandez B, Strootman D. The prostacyclin analog, treprostinil sodium, provides symptom relief in severe Buerger's disease--a case report and review of literature. Angiology. 2006; 57: 99-102.

8 Drake ME. Winiwarter-Buerger disease ('thromboangiitis obliterans') with cerebral involvement. JAMA. 1982; 248: 1870-2.

9 Campello Morer I, Capablo Liesa JL, Guelbenzu Morte S, Carod Artal J, López Gastón Jl, Ara Callizo JR. [Thromboangiitis obliterans with cerebral involvement]. Neurologia. 1995; 10: 384-6.

10 LIPPMANN HI. Cerebrovascular thrombosis in patients with Buerger's disease. Circulation. 1952; 5: 680-92.

11 Calgüneri M, Oztürk MA, Ay H, Arsava EM, Altinok $\mathrm{D}$, Ertenli I, et al. Buerger's disease with multisystem involvement. A case report and a review of the literature. Angiology. 2004; 55: 325-8.

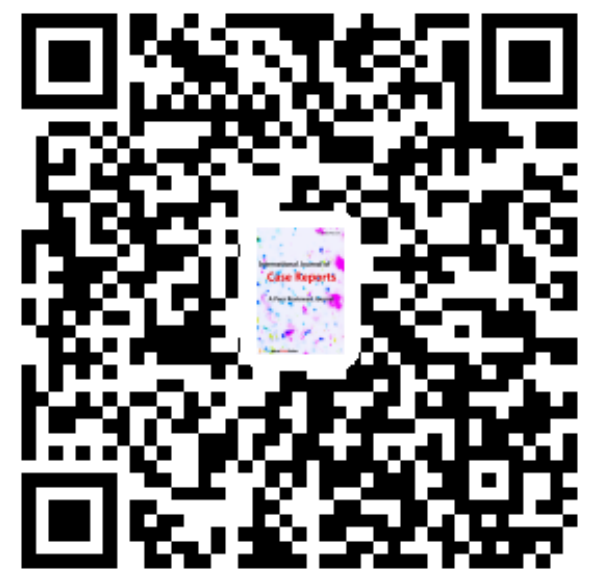

